\title{
Estrategias didácticas en el aprendizaje de las operaciones de polinomio con el uso de la geometría
}

\author{
Didactic strategies in the learning of polynomial \\ operations with the use of geometry
}

Rosa María Castillo ${ }^{1}$

Eugenio Casimiro López Mairena ${ }^{2}$

\section{Resumen}

En la presente investigación, se abordó el uso de la geometría y la manipulación de material concreto como una herramienta pedagógica para la enseñanza de operaciones con polinomios, mediante la implementación de juegos algebraicos, que permitió la dinamización de las clases bajo la metodología activa participativa. Se practicaron variadas estrategias metodológicas, entre ellas: el rompecabezas algebraico, el dominó algebraico, la caja de polinomios mediante el uso de teselas para representar divisiones de polinomios, lo que permitió la consolidación de dichas operaciones. Se concluye, que la vinculación de la variedad de estrategias generó un estado de ánimo satisfactorio por parte de los participantes, creando conciencia en los estudiantes de Física-Matemática sobre la importancia de manipular material concreto en el desarrollo de distintos temas que, a pesar de su grado de dificultad, puede haber maneras de ser adaptado e innovar formas de utilizarlo.

Palabras clave: Juegos algebraicos; Estrategias; Herramientas pedagógicas; Aprendizaje significativo

\section{Abstract}

In the present investigation, the use of Geometry and the manipulation of concrete material as a pedagogical tool for the teaching of operations with polynomials was addressed, through the implementation of algebraic games, which allowed the dynamization of the classes under the active participatory methodology. Several methodological strategies were practiced, among them: the algebraic puzzle, the algebraic domino, the polynomial box by using tesserae to represent polynomial divisions, which allowed the consolidation of these operations. In conclusion, it is affirmed that the linking of the variety of strategies generated a satisfactory state of mind on the part of the participants, creating awareness in the students of Physics-Mathematics on the importance of manipulating concrete material in the development of different

1 Doctora en Matemáticas Aplicadas. Profesora de la Bluefields Indian and Caribbean University-Recinto Universitario El Rama. Correo: roma16o373@yahoo.es

2 Doctor en Innovación en la formación. Vicerrector de la Universidad de las Regiones Autónomas de la Costa Caribe NicaragüenseRecinto Universitario de Nueva Guinea. Correo: eugenio.lopez@uraccan.edu.ni ORCID: https://orcid.org/oooo-0002-7929-7817

Recibido: 01/11/2017 Aprobado: 01/01/2018

Castillo, R., \& López-Mairena, E. (2018). Estrategias didácticas en el aprendizaje de las operaciones de polinomio con el uso de la geometría. Revista Electrónica de Conocimientos, Saberes y Prácticas, 1(1), 28-41. D0I: https://doi.org/10.30698/recsp.v111.2 
subjects that, despite from your degree of difficulty, there may be ways to be adapted and innovate ways to use it.

Key Words: Algebraic games; Strategies; pedagogical tools; meaningful learning;

\section{Introducción}

El siguiente trabajo investigativo está orientado a toda la comunidad educativa que tenga el interés de experimentar estrategias de enseñanza-aprendizaje en las operaciones con polinomios mediante el uso de la geometría como una herramienta didáctica. Se pretende hacer un análisis profundo sobre las diferentes propuestas que se han venido realizando en el transcurso del tiempo y además se propone una intervención pedagógica para el tratamiento metodológico de dicha temática. El objetivo principal de esta investigación es contribuir en la formación pedagógica de docentes de Matemática, a través de la elaboración de propuestas didácticas que fomenten el aprendizaje de las operaciones con polinomios, tomando como herramienta principal el uso de la Geometría Plana, mediante variadas estrategias de enseñanza-aprendizaje con el uso de materiales concretos.

En este sentido, se proponen interrogantes que debe responder el profesorado, esto permitirá hacer una reflexión sobre la práctica docente como las siguientes:

- ¿Qué importancia tiene el uso de conceptos geométricos para el diseño de estrategias didácticas en el proceso enseñanza-aprendizaje de las operaciones con polinomios?

- ¿Qué estrategias didácticas utiliza el docente en el proceso enseñanza- aprendizaje de operaciones con polinomios?

- ¿De qué manera el docente hace uso de la Geometría en la enseñanza del contenido de operaciones con polinomios?

- ¿Cómo elaborar una propuesta didáctica que fomente la formación pedagógica de docentes de Matemática en la enseñanza de las operaciones con polinomios?

- ¿De qué manera se puede lograr una mayor motivación de los estudiantes en la enseñanza de las operaciones con polinomios?

- ¿Cómo lograr que el contenido de las operaciones de polinomios se transforme en actividades tangibles?

- ¿Cómo alcanzar un aprendizaje socialmente útil en los estudiantes si se implementan estrategias de enseñanza en las operaciones con polinomios?

En la búsqueda de respuestas a las interrogantes anteriores, se procedió a realizar la intervención pedagógica mediante la metodología de una investigación-acción para la validación de las diferentes estrategias, dar a conocer todo el proceso, sus logros y dificultades que se experimentaron durante el desarrollo de las distintas acciones 
con los estudiantes de primer año de la carrera de Física- Matemática en la Bluefields Indian \& Caribbean University de la Costa Caribe Sur Nicaragüense.

\section{Literatura}

Esta propuesta es sustentada dentro de dos aspectos fundamentales (Estrategias de enseñanza y su posible tratamiento metodológico) que le dan soporte cognitivo, didáctico y matemático, así como las principales categorías que lo estructuran. De cada una de ellas, se hace mención y un análisis de las diferentes ideas, entre ellas se pueden mencionar algunas como las siguientes:

\section{¿Qué es una estrategia didáctica y qué papel desempeñan en el proceso enseñanza-aprendizaje?}

En el campo educativo han sido muchas las definiciones que se han propuesto para explicar este concepto, que han sido concebidas de diferentes visiones y a partir de diferentes aspectos. A continuación, se hace mención de algunos conceptos:

Las estrategias de enseñanza son los métodos, técnicas, procedimientos y recursos que se planifican de acuerdo con las necesidades de la población a la cual va dirigida y que tiene por objeto hacer más efectivo el proceso de enseñanza-aprendizaje. (Bondy, 2004). Las estrategias de aprendizaje son secuencias de procedimientos o planes orientados hacia la consecución de metas de aprendizaje, mientras que los procedimientos específicos dentro de esa secuencia se denominan tácticas de aprendizaje (Schunk, 1991). El concepto de estrategias didácticas se involucra con la selección de actividades y prácticas pedagógicas en diferentes momentos formativos, métodos y recursos de la docencia. Son acciones planificadas por el docente con el objetivo de que el estudiante logre la construcción del aprendizaje y se alcancen los objetivos planteados. (Lara, 2013).

No se puede desligar la estrategia didáctica de la técnica, sus pasos definen claramente cómo ha de ser guiado el curso de las acciones para conseguir los objetivos propuestos. Es el procedimiento lógico y con fundamento psicológico destinado a orientar el aprendizaje del alumno. En fin, las estrategias didácticas, las técnicas, los métodos y la implementación de metodologías activas, participativas permitirán al docente el logro de los objetivos propuestos. Para ello se debe alcanzar la motivación de los estudiantes, de tal manera que genere confianza, mucho interés de aprender, a través de ambientes pedagógicos agradables y que apunten a cambios de conductas positivas en pro de un aprendizaje significativo. 


\section{¿Cómo obtener un aprendizaje significativo?}

De acuerdo con los razonamientos que se han venido realizando, podemos referirnos que el buen uso e implementación de las estrategias didácticas nos conllevan a un aprendizaje significativo. Dicho fundamento se puede relacionar con la teoría de David Ausubel citado por Solís (2014) quien manifiesta que la esencia del proceso de aprendizaje significativo, es que las ideas expresadas simbólicamente se relacionen de manera sustantiva con conocimientos previos del individuo. Resulta oportuno mencionar la teoría de Ausubel citado por Tünnermann (2011), en su artículo "El constructivismo y el aprendizaje de los estudiantes" quien define condiciones básicas para que se produzca el aprendizaje significativo como los materiales de enseñanza, su organización retomando estilos de aprendizaje de los estudiantes y la motivación. He ahí la importancia de una buena selección de las estrategias de enseñanza-aprendizaje, retomando conocimientos previos de los estudiantes, así como sus diferencias individuales. En este sentido se complementa con el papel del docente en dicho proceso y las condiciones de un excelente ambiente pedagógico.

\section{Reflexión sobre la importancia de estrategias para la enseñanza y el aprendizaje del álgebra.}

Las estrategias de enseñanza aprendizaje ayudan al auto-aprendizaje del estudiante a través de la actividad basándose en sus facultades físicas y mentales, siempre que el docente se convierta en orientador y facilitador tomando en cuenta que lo principal es que el estudiante sea capaz de descubrir por sí mismo sus propios conocimientos, retomando experiencias del contexto que le permitan explorar, descubrir situaciones basadas en sus propios intereses y necesidades. Se debe reconocer el impacto positivo que generan las buenas prácticas de las estrategias de enseñanza en los educandos y los resultados que se pueden obtener a favor del proceso enseñanza-aprendizaje, situación que nos debe llevar a reflexionar y cambiar nuestras prácticas educativas.

Es necesario retomar las nuevas exigencias de la demanda estudiantil, así como del sistema educativo para Educación Media, con el principal objetivo que es elevar la calidad de la educación, que responda a las necesidades del desarrollo humano y científico, tal y como lo plantea Artigue (2004) quien hace énfasis en el desarrollo tecnológico como herramienta básica para enseñar Matemática.

\section{Metodología de investigación}

\section{Línea de la investigación}

La línea de investigación es socio educativa, ya que es propia de las Ciencias Sociales y de este tipo de variable cualitativa, además de la interacción directa con los protagonistas de dicha investigación, desde su fase inicial mediante un diagnóstico que permitió 
visualizar la necesidad o problemática con respecto al tratamiento metodológico en el proceso enseñanza-aprendizaje del Álgebra y su relación con la Geometría. Tal y como lo plantea Gurdián (2007) en su libro: El Paradigma Cualitativo en la Investigación Socio-Educativa de la Colección Investigación y Desarrollo Educativo Regional (IDER), donde hace énfasis que la investigación en este paradigma debe buscar soluciones a los problemas educativos, así como la búsqueda de mejorar las prácticas educativas, el entendimiento de las mismas y las situaciones sociales dentro de las cuales tienen lugar los participantes.

\section{Alcance de la investigación}

Esta investigación es un estudio correlacional, debido que está sustentada bajo la relación que existe entre las variables en estudio como: enseñanza y aprendizaje; es decir, se pretende medir la relación que existe en el proceso enseñanza-aprendizaje al aplicar estrategias de enseñanza y poder medir los resultados que apuntan hacia un aprendizaje significativo, más duradero y de mejor calidad. En este caso se evaluó el grado de asociación entre la enseñanza y el aprendizaje de las operaciones entre polinomios, así como la incidencia en el uso de estrategias didácticas, lo que admite cuantificar y analizar dicha vinculación, a través de la intervención pedagógica.

\section{Métodos de la investigación}

Los métodos utilizados en esta investigación fue una combinación de los mismos, que permitieron una sistematización de los resultados y que se describen a continuación: el método analítico, debido que se realizó un análisis profundo de la temática en estudio enfocado a las estrategias de enseñanza relacionadas con las operaciones de polinomios, así como la realización de una revisión ordenada y exhaustiva de las diferentes propuestas planteadas concernientes con el estudio de este caso.

Se considera también la aplicación del método histórico lógico debido a la descripción histórica y la evolución en este caso que ha tenido la enseñanza de las Matemática, desde la aplicación de la Geometría por parte de los griegos en la antigüedad y su relación actual que tiene con el Álgebra, así como la necesidad de ir implementando nuevas estrategias que respondan a los intereses de las recientes generaciones que cada día se apropian del desarrollo de la tecnología y que debe ser aprovechada como una herramienta más en el desarrollo de la Didáctica de las Matemáticas.

También se retomaron aspectos necesarios del método crítico debido a la naturaleza de la investigación que implica una reflexión de la realidad y generar nuevos cambios de forma consciente, tal y como lo plantea Balandra \& Álvarez (2014), quienes manifiestan que la realidad es un proceso continuo y de permanente cambio, que sólo se puede conocer a través del pensamiento en forma de abstracción para traducirse en práctica que transforma (p. 43). 
Finalmente se consideró también la aplicación del método experimental, debido que mediante el análisis profundo de las diferentes estrategias que se han venido proponiendo y aplicando por otros autores, se retomaron insumos de éstas y fueron contextualizadas para proponer nuevas estrategias novedosas tanto para el que aprende como para el que enseña. En este sentido se generó un ambiente favorable en la búsqueda consciente de emprender nuevas prácticas pedagógicas por parte de los participantes de dicha investigación educativa.

\section{Instrumentos y técnicas utilizadas}

Los instrumentos que se aplicaron en esta investigación, son propios de variable cualitativa, producto del perfil de dicha propuesta como:

- La entrevista en profundidad o no estructurada: Instrumento que permitió que el entrevistado diera su opinión e interpretar esa visión particular de la temática en estudio, así también, el relato de su experiencia o situaciones vividas relacionadas con el asunto en estudio.

- La entrevista estructurada, también denominada directiva, formal o estandarizada, que permitió la recolección directa de la información sobre la base de un formulario que garantizó el control de las posibles respuestas ya que se elaboraron las preguntas sobre la base de un mismo orden y en los mismos términos para las personas entrevistadas.

- Con relación a las técnicas que se implementaron durante todo el proceso y desarrollo de esta investigación, se retomó la observación directa y la observación participante, lo que permitió la realización de un análisis real, la síntesis y la apropiada interpretación del objeto en estudio.

- El diario de campo fue un instrumento básico en este proceso investigativo, ya que permitió realizar una narración minuciosa de los hechos registrados en el cuaderno de notas y sirvieron de insumos para retomar todos aquellos aspectos que le dieron rigor y carácter científico a dicha investigación.

\section{Población y muestra}

El universo se conformó por todos los estudiantes de las diferentes carreras de Ciencia de la Educación en la Bluefields Indian \& Caribbean University (BICU), sede Rama cuya matrícula corresponde a 213, con una población de 60 estudiantes y una muestra de 42 estudiantes de la carrera de Física-Matemática, inscritos en primer año de dicha universidad que corresponde al 70\% de la población. 


\section{Resultados}

\section{Análisis sobre la situación actual del tratamiento metodológico en la enseñanza de operaciones con polinomios}

Se realizó una breve exploración diagnóstica con los docentes que atienden matemática en la secundaria, lo que permitió obtener una radiografía de la situación actual que a continuación se describe:

Una de las dificultades planteada por los docentes en la enseñanza de operaciones con polinomios es la ausencia de estrategias didácticas que abarquen los diferentes algoritmos de las operaciones con polinomios, se siente el divorcio entre el Álgebra y la Aritmética, los estudiantes presentan muchas dificultades al establecer el tránsito de la Aritmética al Álgebra debido a la falta de dominio de operaciones básicas especialmente con números racionales. Es bien común la problemática de la falta de manejo de la ley de los signos por parte de un buen número de estudiantes debido que confunden la ley de los signos de la suma con la multiplicación, lo que viene a repercutir en las diferentes operaciones algebraicas y de paso de acuerdo a la valoración de los docentes hay poca aceptación por parte de los estudiantes sobre el uso de variables algebraicas.

Según los docentes existen otros factores externos que también afectan de forma indirecta el aprendizaje del Álgebra en secundaria y se debe a la falta de acompañamiento de los padres de familia para con sus hijos, que de alguna manera suma a la falta de interés por aprender. Otro factor importante que señalan los docentes y que ha venido a incidir negativamente en el dominio de operaciones algebraicas, es la falta de dominio de las tablas de multiplicar por parte de un grupo considerable de estudiantes, a pesar que en ocasiones se han realizado juegos didácticos para alcanzar el dominio de las mismas.

Cabe señalar que en la mayoría de los casos los únicos medios didácticos utilizados son los libros de textos que actualmente existen en la Educación Secundaria en Nicaragua y que han sido de gran apoyo especialmente en las zonas rurales, debido que no se cuenta con energía eléctrica para poder disponer de otras alternativas. Sin embargo, se han venido haciendo algunos esfuerzos por parte de los docentes haciendo uso de papelógrafos, la elaboración de murales informativos respecto a la temática en estudio. El papel de los alumnos monitores ha sido muy relevante, colaborando con el docente en la atención individualizada de los estudiantes que presentan mayores dificultades.

Con relación a la implementación de software matemático u otras herramientas tecnológicas en las clases de Matemática ha sido mínima por parte de los docentes, debido a la falta de dichos medios tecnológicos y a pesar que se han venido dotando 
algunos laboratorios de computación en los institutos más grandes en el municipio, aún no hay suficientes condiciones para su implementación. Sin embargo, algunos estudiantes hacen uso de ciertas aplicaciones en sus teléfonos inteligentes con sistema operativo Android ${ }^{3}$ como MalMath ${ }^{4}$ entre otros.

En general, los docentes consideran la necesidad de capacitaciones metodológicas sobre estrategias de enseñanza para el aprendizaje de las Matemáticas especialmente en la enseñanza del Álgebra haciendo énfasis en las operaciones con polinomios, retomando que es una unidad nueva para los estudiantes y que es notorio el salto que experimentan los estudiantes de operaciones aritméticas a operaciones algebraicas. Se valora de gran relevancia la idea de la propuesta metodológica, en la búsqueda de mejorar y fortalecer el escenario pedagógico tanto para docentes como para estudiantes, ya que las consecuencias causadas por las dificultades en la comprensión de las operaciones con polinomios contribuyen a la desmotivación de los estudiantes y lo más preocupante es la deficiencia que presentan éstos en los siguientes niveles académicos.

\section{Algunos insumos sobre la intervención pedagógica implementada}

Se utilizaron algunos conceptos geométricos como: el polígono estrellado para la consolidación de la suma de polinomio, así como cálculo de regiones sombreadas enfocadas a la resta de polinomio y su comprobación aritmética mediante la asignación de valores a las variables. El uso de teselas (fichas elaboradas con cartulinas de colores) permitió el desarrollo de la multiplicación, así como el uso de rompecabezas y el dominó algebraico. La caja polinomio permitió la manipulación de material concreto y la comprobación de divisiones exactas de polinomios. Todo lo anterior fue implementado mediante el desarrollo de ocho acciones que contienen las actividades implementadas y su debida hoja de trabajo para uso personal del estudiante.

Se implementaron ocho acciones acompañadas de momentos didácticos alusivos a la manipulación de material concreto mediante las diferentes estrategias aplicadas como:

- Uso de teselas algebraicas o tabletas algebraicas

- El dominó algebraico

- El rompecabezas algebraico

- Figuras geométricas planas

3 Android es un sistema operativo basado en el núcleo Linux. Fue diseñado principalmente para dispositivos móviles con pantalla táctil, como teléfonos inteligentes, tabletas, entre otros.

4 MalMath aplicación para el sistema Android es un solucionador de problemas de matemáticas con descripción paso a paso y la vista gráfica. La aplicación, que es gratuita y funciona sin necesidad de internet, permite llevar a cabo operaciones como integrales, derivadas, límites, ecuaciones simples o trigonometría variada. 
La intervención pedagógica se desarrolló aplicando una metodología participativa mediante trabajos en equipos, en la cual se aplicaron las ocho guías de trabajo, desarrolladas en dos momentos:

- Momento práctico con material concreto elaborado con cartulinas de colores, donde los estudiantes tuvieron la oportunidad de realizar las diferentes actividades que conllevaron a obtener resultados algebraicos de las diferentes operaciones de polinomios y que permitió enlazar con la parte procedimental.

- Momento práctico mediante el afianzamiento de algoritmos y procedimientos algebraicos que recogieron los aspectos que integran las estructuras de las operaciones con polinomios enlazadas con los diferentes procesos aritméticos al ir comprobando áreas y perímetros de las figuras dadas a través de la asignación de números enteros.

Hubo una buena aceptación de todas las actividades realizadas por parte de los estudiantes, lo que permitió hacer conciencia de las ventajas que genera la implementación de variadas estrategias metodológicas en el proceso enseñanza-aprendizaje del Álgebra.

\section{Validación de las acciones pedagógicas implementadas}

a) Valoración de las actividades realizadas en las diferentes operaciones con polinomios

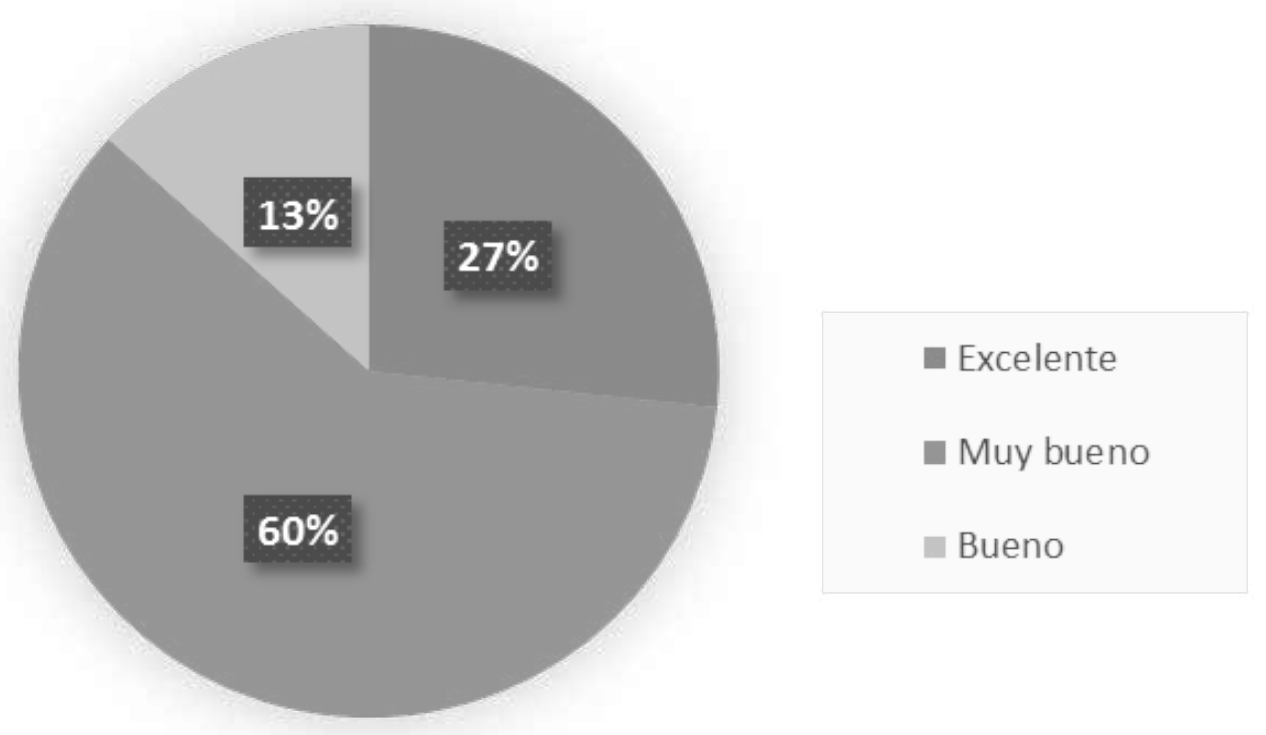

Figura 1: Valoración de las actividades didácticas implementadas 
El 60\% de los estudiantes de Física-Matemática consideran muy buena la propuesta didáctica, debido a la facilidad que proporciona para aprender de forma más rápida y con un buen grado de comprensión, así como despertar mayor interés por los diferentes juegos realizados, permitiendo la asimilación de forma rápida debido al contacto con materiales concretos.

\section{b) Evaluación de los materiales concretos utilizados durante la intervención pedagógica}

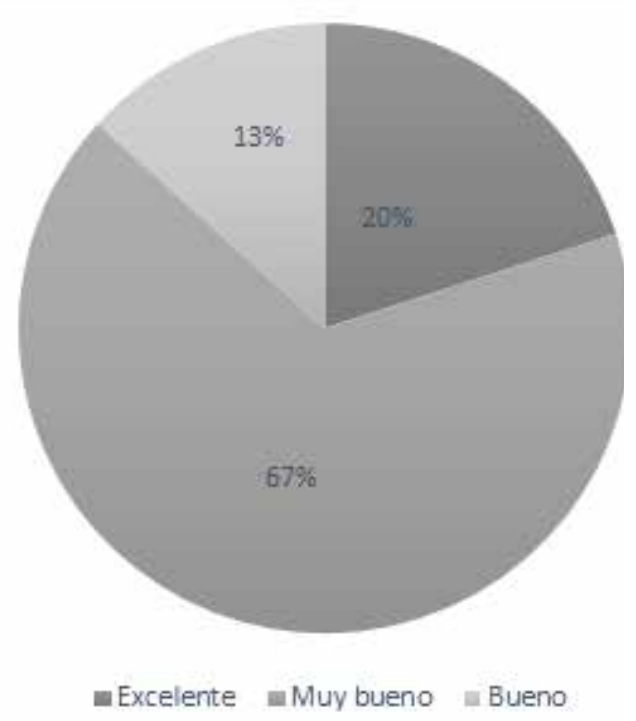

Figura 2: Materiales didácticos utilizados

El 67\% de los estudiantes consideran muy importante la utilización de materiales concretos durante la implementación de las ocho intervenciones didácticas debido a las diferentes ventajas que representa la utilización de los mismos y manifestaron que permite la consolidación de valores como ser solidarios, respetuosos y honestos. El haber integrado cálculo de áreas y perímetros en operaciones algebraicas contribuyó a la dinamización de las diferentes actividades despertando un mayor interés, así como establecer relaciones con escenarios de la vida cotidiana lo que permitió generar conciencia sobre la utilidad de las variables en muchas ocasiones. También, al manipular materiales concretos se considera una excelente oportunidad para el desarrollo del pensamiento lógico de los estudiantes ya que despierta curiosidad y a través de las construcciones realizadas permitió armar los diferentes rompecabezas donde se logró observar la consolidación del trabajo en equipos. 


\section{Discusión y conclusiones}

En este estudio revela la realidad a cerca del tratamiento metodológico sobre las operaciones con polinomios en el municipio de El Rama, Región Autónoma de la Costa Caribe Sur Nicaragüense (RACCS), tomando como referencia los docentes de Matemática en el nivel de Secundaria.

Dicho proceso se valida con el diagnóstico inicial que de forma generalizada se confirma la poca aplicación de estrategias didácticas relacionada con la temática en estudio, así como la falta de recursos didácticos en las clases de Matemática, además de la no relación de Geometría con operaciones de polinomios, solamente cuando se desarrolla la temática de ecuaciones lineales que se aplican en algunos temas como: ángulos internos y externos de un triángulo, teorema de Thales, teorema de Pitágoras, entre otros.

En vista de la verificación sobre la carencia en la aplicación de estrategias novedosas relacionadas con las operaciones con polinomios, se considera que dicha propuesta didáctica será de mucha utilidad y permitirá a los docentes la pauta para seguir innovando nuevas acciones en la búsqueda de aprendizajes más significativos, que despierten el interés en los estudiantes e incentiven el amor hacia las matemáticas.

Se logró experimentar directamente la aplicación de estrategias didácticas en el proceso de enseñanza-aprendizaje de las operaciones con polinomios a través del uso de la Geometría. En ese sentido, podríamos afirmar, en cierta medida, que los resultados obtenidos son óptimos, debido a la concatenación de la parte teórica con la práctica y su complementación mediante el uso y manipulación de materiales didácticos concretos.

La vinculación de la variedad de estrategias generó un estado de ánimo satisfactorio por parte de los participantes, creando conciencia en los estudiantes de FísicaMatemática sobre la importancia de manipular material concreto en el desarrollo de distintos temas que, a pesar de su grado de dificultad, puede haber maneras de ser adaptado e innovar formas de utilizarlo. La utilización de estrategias de enseñanza mediante juegos algebraicos y la implementación de figuras geométricas permitieron la representación de operaciones con polinomios, favoreciendo el aprendizaje activo y participativo, así como una mejor actitud hacia la Matemática por parte de los estudiantes. Por tales razones, se puede afirmar que en todo el proceso de las diferentes operaciones con polinomios resultó de gran utilidad el material didáctico del Álgebra geométrica, acompañado de guías didácticas que fomentaron el trabajo colaborativo en los diferentes equipos, permitiendo el fortalecimiento de valores en cada estudiante.

Finalmente, las actividades realizadas como el uso de teselas, la caja de polinomios, el rompe cabezas y el dominó algebraico, además de las operaciones con polinomios, 
permite oportunidades para ser utilizado en otros temas algebraicos como factorización y productos notables, donde las operaciones con polinomios sientan las bases para el dominio de los temas antes mencionados, por lo que dichas estrategias implementadas pueden ser generalizadas hacia conocimientos más amplios.

\section{Perspectivas futuras}

En vista de la relevancia que representa la capacitación del profesorado de Matemática para un mejor desempeño docente y que influya en el buen aprendizaje de los estudiantes, se sugiere que las autoridades correspondientes de la BICU, coordinen con el Ministerio de Educación para incluir dicha propuesta en el desarrollo de los EPI (Encuentros Pedagógicos de Inter aprendizaje).

El docente debe ser innovador de estrategias de enseñanza de forma permanente, por lo tanto, se recomienda a todos los docentes que hagan uso de dicha propuesta didáctica y que experimenten nuevas actividades. Se sugiere que los docentes de secundaria experimenten el tratamiento metodológico de otras temáticas de la enseñanza del Álgebra tomando como insumo dicha propuesta didáctica, ya que puede ser adaptada a productos notables y factorización, entre otros.

Se desea que se genere una mejora continua de esta propuesta didáctica, debido a los resultados positivos obtenidos durante la aplicación de la misma; es por eso que se recomienda a todos los docentes investigadores, interesados en retomar información, la puedan mejorar y aportar nuevos insumos en pro de la preparación de las nuevas generaciones. Si se cuenta con laboratorios de computación, se sugiere reforzar los conocimientos adquiridos por los estudiantes sobre las operaciones con polinomios a través del uso de software matemático.

Además, se recomienda enseñar a los estudiantes a utilizar aplicaciones como MalMath en sus teléfonos inteligentes para que les sirva como un medio de comprobación de resultados.

\section{Lista de referencias}

Ballén, J. O. (2012). Obtenido de El álgebra geométrica como recurso didáctico para la factorización de polinomios de segundo grado:: http://www.bdigital.unal.edu. co/8063/1/javierorlandoball\%C3\%Agnnovoa.pdf

Bouzas, P. G. (og de marzo de 2009). www.investigacionenlaescuela.es. Obtenido de Dificultades en el paso de la aritmética al álgebra escolar: ¿Puede ayudar el aprendizaje cooperativo?: http://www.investigacionenlaescuela.es/articulos/R73/R73.7.pdf 
Campuzano, W. A. (2016). Transición aritmética al algebra en la factorización de expresiones algebraicas. Obtenido de http://ayura.udea.edu.co:8080/jspui/bitstream/123456789/2507/1/JCo274_walteravilezcampuzano.pdf

Castillo, R. M. (noviembre de 2013). Obtenido de Estrategias de enseñanza para el aprendizaje de operaciones con polinomios de octavo grado en el colegio Violeta Barrios de Chamorro, comunidad Wapy, municipio El Rama.

Díaz, F., \& Hernández, G. (2002). Obtenido de Estrategias docentes para un aprendizaje significativo. Una interpretación constructivista.: http://memsupn.weebly.com/ uploads/6/o/o/7/60077005/estrategias_docentes_para_un_apje._significativofrida_d\%C3\%8Daz_barriga_parte_1_de_4.pdf

Fernández, W. (2013). Diseño e implementación de una propuesta didáctica de enseñanza de las operaciones algebraicas de adición y sustracción, articulando la transición de la aritmética al álgebra en el $8^{\circ} \mathrm{A}$ de la institución educativa Las nieves. Obtenido de http://www.bdigital.unal.edu.co/11782/1/85167251.2014.pdf

Huertas, E. Y. (30 de noviembre de 2011). ¿En qué medida las estrategias metodológicas influyen en el aprendizaje de las operaciones con polinomios? Obtenido de http:// www.monografias.com/trabajos62/estrategias-metodologicas-aprendizajepolinomios/estrategias-metodologicas-aprendizaje-polinomios2.shtml

Jiménez, S. M. (diciembre de 2013). Tabletas algebraicas como una alternativa de enseñanza del proceso de factorización de algunos polinomios de segundo grado. Obtenido de http://repositorio.pedagogica.edu.co/xmlui/bitstream/handle/123456789/474/ TE-16459.pdf?sequence=1

Morales, C. G. (2014). Obtenido de Estrategias metodológicas para contribuir el aprendizaje del Algebra y Geometría en el primero de bachillerato del colegio nacional mixto San Joaquín durante el año lectivo 2012-2013.: http://dspace.ucuenca.edu. ec/bitstream/123456789/20836/1/Tesis.pdf

Osorio, M. (2016). El paso de la aritmética al álgebra. Obtenido de http://www.bdigital. unal.edu.co/56283/1/7709140.2017.pdf

Parajón, R. A. (27 de septiembre de 2009). Álgebra, su tratamiento pedagógico y aplicaciones. Managua, Nicaragua.

Salazar, V. P., Jiménez, S. M., \& Mora, L. C. (o6 de noviembre de 2013). Obtenido de Tabletas algebraicas, una alternativa de enseñanza del proceso de factorización.: http://www.centroedumatematica.com/memorias-icemacyc/356-520-1-DR-T. pdf 
Salazar, W. H. (2016). Obtenido de Enseñanza de los conceptos perímetros, áreas y volumen a estudiantes de sexto grado, a partir de maquetas.: http://www.bdigital.unal.edu. co/51465/1/7700751.2016.pdf

Sandoval, Y. E. (29 de noviembre de 2010). Obtenido de Las representaciones geométricas como herramienta para la construcción del significado de expresiones y operaciones algebraicas, desarrollado con alumnos de octavo grado del instituto "San José del Pedregal»: http://biblioteca.upnfm.edu.hn/images/tesis\%2oclasificadas/ Maestr\% C3\%ADa\%2oen\%2oMatem\%C3\%A1tica\%2oEducativa/yelsin_sandoval.pdf

Soto, F., Mosquera, S., \& Gómez, C. (1 de junio de 2005). Obtenido de La caja de polinomios: http://revistaerm.univalle.edu.co/VolXIIIN1/mosquera.pdf

Tünnermann Bernheim, C. (marzo de 2011). www.redalyc.org. Obtenido de El constructivismo y el aprendizaje de los estudiantes.: http://www.redalyc.org/ pdf/373/37319199005.pdf

Villarroel, J. M. (2014). Obtenido de Propuesta para la enseñanza de las operaciones de polinomios y el proceso de factorización, con la herramienta didáctica "caja de polinomios»: http://www.bdigital.unal.edu.co/45981/1/1085249161.2014.pdf 